\title{
Publications Received for Review
}

Phonetica 2000;57:75-78

Each fascicle of Phonetica provides a list of the publications that have been received for review. Readers who would like to write critical commentaries on any of these books for Phonetica are requested to contact the editor,

Prof. K.J. Kohler, Institut für Phonetik und digitale Sprachverarbeitung, Universität Kiel, D-24098 Kiel, FRG,

who will arrange for the copies to be sent to them. At the same time, he will inform them about the expected length of the review as well as other technical details, and suggest a date for submitting the manuscripts. The time allotted for preparing reviews will on principle be kept as short as possible in order to enable Phonetica to fulfill its obligation of keeping its readers up to date with publications in the field of speech science. Readers are also welcome to suggest any other book in our field for review in Phonetica, over and above the ones named in the list of received publications. The editor will then take the necessary steps to obtain copies from the publishing firms.

Archangeli, Diana, Langendoen, D. Terence (eds): Optimality Theory. 233 pp.; £45.(hbk), £ 15.99 (pbk); Blackwell 1997; ISBN 0-631-20226-9.

Ayuso, Antonio J. Rubio, Soler, Juan M. López: Speech Recognition and Coding. New Advances and Trends. Computer and Systems Sciences, Vol. 147. Springer 1995, XI + 505 pp.; DM 188.-; ISBN 3-540-60098-1.

Martin J. Ball, Raymond D. Kent: The New Phonologies - Developments in Clinical Linguistics, 272 pp.; Singular Publishing Group, Inc., 1997; ISBN 1-56593-082-7.
Martin J. Ball, Joan Rahilly: Phonetics. The Science of Speech, 239 pp.; £14.99. Arnold 1999, ISBN 0-340-70009-2.

Braun, Angelika, \& Köster, Jens-Peter (eds.): Studies in Forensic Phonetics. Wissenschaftlicher Verlag Trier 1995, 167 pp.; DM 36.-; ISBN 3-88476-150-1.

Braun, Angelika (Hrsg.): Untersuchungen zu Stimme und Sprache. Papers on Speech and Voice (= Zeitschrift für Dialektologie und Linguistik, Beihefte 96). Franz Steiner, Stuttgart 1996, 166 pp.; DM/SFr. 76.-(öS 555.-); ISBN 3-515-07025-7.

\section{KARGER}

Fax + 41613061234 E-Mail karger@karger.ch www.karger.com 
Braun, Angelika (ed.): Advances in Phonetics. Proceedings of the International Phonetic Sciences Conference (IPS) Bellingham, Wa., June 27-30, 1998. ZDL Beihelfte 106. Franz Steiner Verlag Stuttgart, 1999; ISBN 3-515-07655-7.

Breitung, Horst: Phonetik - Intonation Kommunikation. 2 Standpunkte zur Sprachund Kulturvermittlung. Goethe Institut, München 1994, 224 pp.; ISBN 3-930220-01-6.

Chin, Steven B, Pisoni, David B: Alcohol and Speech. 362 pp.; \$49.95, Academic Press 1997; ISBN 0-12-172775-0.

Clausnitzer Volkmar, Clausnitzer Renate (Hrsg.): Logopädie für Studierende und Praktiker, Band 1. Grundlagen der Therapie von Sprach- und Sprechstörungen. Hüthig 1997, XV + 390 pp.; DM 98.-, SFr. 89.-; ISBN 3-7785-2361-9.

Coleman, John: Phonological Representations: Their Names, Formes and Powers. Cambridge University Press. £50.- (hbk.); ISBN 0-521-47208-3.

Collins, Beverley, Mees, Inger M.: The Real Professor Higgins. The Life and Career of Daiel Jones, 572 pp.; DM 248.-, W. de Gruyter 1998; ISBN 3-11-015124-3.

Connell, A., Arvaniti, A.: Phonology and Phonetic Evidence. Paper in Phonology 4. Cambridge University Press 1995, 403 pp.; £40.-; ISBN 0-521-48259-3.

Cruttenden, Alan: Intonation. 2nd edition, Cambridge Textbooks in Linguistics: 201 pp.; $£$ 13.95, Cambridge Uni Press 1997; ISBN 0-521-59825-7.

Davenport, Mike, Hannahs, S.J.: Introducing Phonetics and Phonology; 196 pp.; $£ 12.99$, Arnold London, 1998; ISBN 0-340-66217-4.

Davies, Christie: Jokes and their Relation to Society. Humor Research, 234 pp.; DM 178.-, Mouton de Gruyter 1998; ISBN 3-11016104-4.
Davies, Winifred V.: Linguistic Variation and Language Attitudes in Mannheim-Neckarau. Franz Steiner Verlag, Stuttgart 1995, 224 pp.; DM 96.-; ISBN 3-515-06834-1.

Davis, P.J., \& Fletscher, N.H.: Vocal Fold Physiologie. Controlling, Complexity and Chaos. Singular 1996; 433 pp.; £44.95; ISBN 1-56593-714-7.

Dejonckere, P.H., Hirano, M., \& Sundberg, J.: Vibrato. Singular 1995, 152 pp.; £27.50; ISBN 1-56593-146-7.

Paul Foulkes, Gerard Cocherty, Urban Voices: Accent Studies in the British Isles. 314 pp.; £ 16.99, Arnold 1999; ISBN 0-34070607-4.

Fujimura, S., \& Hirano, M.: Vocal Fold Physiology - Voice Quality Control. Singular 1995, 358 pp.; £44.95; ISBN 1-56593-379-6.

Volker Gall, Reni Berg: Feinstrukturen von Stimme und Sprache, inkl. CD-ROM. Wötzel 1998, 89 pp.; DM 298.-; CHF 280.-; ISBN 3-925831-46-0.

Gehrmann, Siegfried: Sprechen als Tätigkeit. Koordinations- und lerntheoretische Grundlagen des zweitsprachligen Ausspracheerwerbs. Reihe Siegen. Beiträge zur Literatur-, Sprachund Medienwissenschaft, 141, 1999, Universitätsverlag C. Winter, Heidelberg; Kt DM 68.-, ATS 496.-, SFr. 60.-; ISBN 3-8253-0867-7.

Grohnfeldt, Manfred (Hrsg.): Störungen der Redefähigkeit. (= Handbuch der Sprachtherapie), 1992, XX, 548 pp.; DM 98.--; ISBN 3-89166-444-3.

Grohnfeldt, Manfred (Hrsg.): Grundlagen der Sprachtherapie. (= Handbuch der Sprachtherapie, Bd. 1), 1996, 2. Aufl., XIII, 356 pp.; DM 64.--; ISBN 3-89166-440-0.

Grohnfeldt, Manfred (Hrsg.): Störungen der Aussprache. (= Handbuch der Sprachtherapie, Bd. 2), 1996, 2. Aufl., XIV, 290 pp.; DM 54.-; ISBN 3-89166-441-9. 
Grohnfeldt, Manfred (Hrsg.): Störungen der Grammatik. (= Handbuch der Sprachtherapie, Bd. 4), 1997, 2. Aufl., XIV, 284 pp.; DM 58.-; ISBN 3-89166-443-5.

Grohnfeldt, Manfred (Hrsg.): Zentrale Sprach- und Sprechstörungen. (= Handbuch der Sprachtherapie, Bd. 6), 1993, XVIII, 531 pp.; DM 98.-; ISBN 3-89166-445-1.

Grohnfeldt, Manfred (Hrsg.): Stimmstörungen. (= Handbuch der Sprachtherapie, Bd. 7), 1994, XVII, 466 pp.; DM 98.-; ISBN 3-89166-446-1.

Carlos Gussenhoven, Haike Jakobs: Understanding Phonology. 286 pp.; £14.99, Arnold 1998; ISBN 0-340-69218-9.

Walter Hulstijn, Hermann F. M. Peters, Pascal H.H.M. van Lieshout: Speech Production: Motor Control, Brain Research and Fluency Disorders, 652 pp.; NLG 442.-; \$276.25; Elsevier Science 1997; ISBN 0-444-82460-X.

Alex F. Johnson, Barbara H. Jacobson: Medical Speech-Language Pathology. A Practioner's Guide, 76 pp.; DM 189.-; G. Thieme 1998; ISBN 3-13-110531-3.

Kent, Raymund, D.: The Speech Sciences. 532 pp., \$47.95; Singular 1997; ISBN 1-56593-689-2.

Kenyon John S.: American Pronunciation. Twelfth edition, expanded. George Wahr Publishing Company 1997, Ann Arbor, Michigan, 410 pp.; ISBN 1-911586-47-4.

Kiritani, Shigeru, Hirose, Hajime, Jujusaki, Hiroya: Speech Production and Language. Speech Research, Vol. 13. 302 pp., DM 188.-, de Gruyter 1997, ISBN 3-11-015277-0.

Krallmann, Dieter, H. Walter Schmitz (Hrsg.): Perspektiven einer Kommunikationswissenschaft, Band 1 und 2. Internationales Gerold Ungeheuer Symposium, Essen 6.-8.7.1995, 556 pp.; Nodus Publikationen Münster, 1998; ISBN 3-89323-651-1, DM 120.-
Ladd, D. Robert: Cambridge Studies in Linguistics, vol. 79. International Phonology. Cambridge Uni. Press 1996, 334 pp.; £14.95; ISBN 0-521-47575-9.

Lehiste, Ilse \& Ross, Jaan: Estonian Prosody: Papers from a Symposium. Institute of Estonian Language, Tallinn 1997, DM 25.-, 277 pp.; ISBN 9985-851-47-1.

Zita McRobbie-Utasi: Quantity in the Skolt (Lappish) Saami Language: An Acoustic Analysis. Uralic and Altaic Series, vol. 165; Denis Sinor (ed); Indiana University, Research Institute for Inner Asian Studies; Bloomington, Indiana, 1999; 225 pp.; \$35.00; ISBN 0-933070-45-4.

Masuya, Yosishiro: Phonetics and Phonology. 214 pp.; Kobian-Shobo Tokyo 1997; ISBN 4-87558-054-1.

Neppert, Joachim M.H.: Elemente einer Akustischen Phonetik. 349 pp.; DM 58.-, Buske Hamburg, 1999; ISBN 3-87548-154-2.

Ode, Cecilia, \& Van Heuven, Vincent J. (eds.): Experimental Studies of Indonesian Prosody (= Semaian 9). Vakgroep Talen en Culturen van Zuidoost-Azië en Oceanië. Rijksuniversitet te Leiden 1994, 214 pp. + VII; Dfl. 40.--; ISBN 90-73084-10-5.

Otake, Takashi, Cutler, Anne: Speech Research 12. Phonological Structure and Language Processing. W. de Gruyter 1996, XII + 254 pp.; DM 158.-; ISBN 3-11-014967-2.

Rischel, Jørgen, \& Basbøll (eds.): Aspects of Danish Prosody, RASK Supplement Vol. 3. Odense University Press 1995, 213 pp.; DKK 200.-; ISBN 87-7838-160-6.

Ross J. Roeser, Donise W. Pearson, Emily A. Tobey: Speech-Language Pathology Desk Reference. 523 pp.; DM 98.-, G. Thieme 1998; ISBN 3-13-110541-0.

Ryalls, Jack: A Basic Introduction to Speech Perception. Singular 1996, 149 pp.; £27.50; ISBN 1-56593-617-5. 
Scheuer, Jann: Tryk på Danske Verber, RASK Supplement Vol. 4. Odense Universitetsforlag 1995, 261 pp.; DKK 200.-; ISBN 87-7838-161-4.

Gilles-Maurice de Schryver: Cilubà Phonetics. Proposals for a 'corpus-based phonetics from below'-approach. Recall Linguistics Series Nr. 14; Recall, Gent 1999; ISBN 9076327-14-9.

Stork, David G., Hennecke, Marcus E. (eds.): Speechreading by Humans and Machines. Models, Systems, and Applications. 1996. XV, 686 pp.; 1185g. Hardcover DM 248.-; $£ 1810.40$; SFr. 216.-; Berlin / Heidelberg / New York / London / Paris / Tokyo / Hong Kong: Springer-Verlag. ISBN 3-540-61264-5. NATO ASI Series.
Van Santen, Jan. P.H., Sproat, Richard W., Olive, Joseph P., Hirschberg, Julia: Progress in Speech Synthesis. Springer 1996, 598 pp.; DM 198.-; ISBN 387-94707-9.

Vieregge, Wilhelm H.: Patho-Symbolphonetik. Auditive Deskription pathologischer Sprache (= Zeitschrift für Dialektologie und Linguistik, Beihefte 100). Franz Steiner, Stuttgart 1996, 239 pp.; DM/SFr. 98.- (öS 715.-); ISBN 3-515-06807-4.

Vihman, Marilyn May: Phonological Development. Blackwell 1996, 312 pp.; \$59.95 (hardback); ISBN 0-631-16353-0, £16.99, \$24.95 (paperback); ISBN 0-631-16354-9. 